\title{
BURNSIDE'S THEOREM FOR HOPF ALGEBRAS
}

\author{
D. S. PASSMAN AND DECLAN QUINN
}

(Communicated by Ken Goodearl)

\begin{abstract}
A classical theorem of Burnside asserts that if $\chi$ is a faithful complex character for the finite group $G$, then every irreducible character of $G$ is a constituent of some power $\chi^{n}$ of $\chi$. Fifty years after this appeared, Steinberg generalized it to a result on semigroup algebras $K[G]$ with $K$ an arbitrary field and with $G$ a semigroup, finite or infinite. Five years later, Rieffel showed that the theorem really concerns bialgebras and Hopf algebras. In this note, we simplify and amplify the latter work.
\end{abstract}

Let $K$ be a field and let $A$ be a $K$-algebra. A map $\Delta: A \rightarrow A \otimes A$ is said to be a comultiplication on $A$ if $\Delta$ is a coassociative $K$-algebra homomorphism. For convenience, we call such a pair $(A, \Delta)$ a b-algebra. Admittedly, this is rather nonstandard notation. One is usually concerned with bialgebras, that is, algebras which are endowed with both a comultiplication $\Delta$ and a counit $\epsilon: A \rightarrow K$. However, semigroup algebras are not bialgebras in general, and the counit rarely comes into play here. Thus it is useful to have a name for this simpler object.

Now a b-algebra homomorphism $\theta: A \rightarrow B$ is an algebra homomorphism which is compatible with the corresponding comultiplications, and the kernel of such a homomorphism is called a b-ideal. It is easy to see that $I$ is a b-ideal of $A$ if and only if $I \triangleleft A$ with $\Delta(I) \subseteq I \otimes A+A \otimes I$. Of course, the b-algebra structure can be used to define the tensor product of $A$-modules. Specifically, if $V$ and $W$ are left $A$-modules, then $A$ acts on $V \otimes W$ via $a(v \otimes w)=\Delta(a)(v \otimes w)$ for all $a \in A, v \in V, w \in W$. Notice that if $I$ is a b-ideal of $A$, then the set of all $A$-modules $V$ with $\operatorname{ann}_{A} V \supseteq I$ is closed under tensor product. Conversely, we have

Proposition 1. Let $A$ be a b-algebra and let $\mathscr{F}$ be a family of A-modules closed under tensor product. Then

$$
I=\bigcap_{V \in \mathscr{F}} \operatorname{ann}_{A} V
$$

is a b-ideal of $A$.

Proof. Certainly $I$ is an ideal of $A$. Now let $X=\oplus \sum_{V \in \mathscr{F}} V$ be the direct sum of the modules in $\mathscr{F}$. Then $X$ is an $A$-module and $\operatorname{ann}_{A} X=\bigcap_{V \in \mathscr{F}} \operatorname{ann}_{A} V=$

Received by the editors February 17, 1993 and, in revised form, April 27, 1993.

1991 Mathematics Subject Classification. Primary 16W30, 16S34, 16S30. 
$I$. Furthermore, since $X \otimes X=\oplus \sum_{V, W \in \mathscr{F}} V \otimes W$ and since each $V \otimes W \in \mathscr{F}$, it follows that $I$ annihilates $X \otimes X$. In other words,

$$
\Delta(I) \subseteq \operatorname{ann}_{A \otimes A} X \otimes X=I \otimes A+A \otimes I
$$

and $I$ is a b-ideal of $A$.

The assumption that $\mathscr{F}$ is closed under tensor product can be weakened somewhat in the above. Indeed, suppose that for each $V, W \in \mathscr{F}$ there exists $U \in \mathscr{F}$ with $\operatorname{ann}_{A} U \subseteq \operatorname{ann}_{A} V \otimes W$. Then certainly $I \subseteq \operatorname{ann}_{A} U$ annihilates $V \otimes W$, so $I$ annihilates $X \otimes X$ and hence $I$ is a b-ideal of $A$.

Now if $(A, \Delta, \epsilon)$ is a bialgebra with counit $\epsilon$, then $I$ is a bi-ideal of $A$ if and only if it is a b-ideal with $\epsilon(I)=0$. Furthermore, we can trivially guarantee that the ideal $I$ of the previous proposition satisfies $\epsilon(I)=0$ by including the principal module $K_{\epsilon}$ in the set $\mathscr{F}$. Thus we have

Proposition 1*. Let A be a bialgebra and let $\mathscr{F}$ be a family of A-modules closed under tensor product. If $K_{\epsilon} \in \mathscr{F}$, then

$$
I=\bigcap_{V \in \mathscr{F}} \operatorname{ann}_{A} V
$$

is a bi-ideal of $A$.

Since the coassociativity of $\Delta$ guarantees that the tensor product of $A$ modules is associative, it makes sense to define the $n$th tensor power of $V$ by

$$
V^{\otimes n}=V \otimes V \otimes \cdots \otimes V \quad(n \text { times })
$$

for all $n \geq 1$. Here, $V^{\otimes 1}=V$ and $V^{\otimes m} \otimes V^{\otimes n}=V^{\otimes(m+n)}$ for all $m, n \geq 1$. It is now a simple matter to prove the following result of [Ri].

Corollary 2. Let $A$ be a b-algebra and let $V$ be an A-module. If $\operatorname{ann}_{A} V$ contains no nonzero b-ideal, then $\mathscr{T}(V)=\oplus \sum_{n=1}^{\infty} V^{\otimes n}$ is a faithful A-module.

Proof. $\mathscr{F}=\left\{V^{\otimes n} \mid n=1,2, \ldots\right\}$ is a set of $A$-modules which is clearly closed under tensor product. Thus, by Proposition 1,

$$
I=\bigcap_{n=1}^{\infty} \operatorname{ann}_{A} V^{\otimes n}=\operatorname{ann}_{A} \mathscr{T}(V)
$$

is a b-ideal of $A$. But $I \subseteq \operatorname{ann}_{A} V^{\otimes 1}=\operatorname{ann}_{A} V$, so the hypothesis implies that $I=0$ and hence that $\mathscr{T}(V)$ is faithful.

If $A$ is a bialgebra, then one usually defines $V^{\otimes 0}$ to equal $K_{\epsilon}$, since the latter module behaves like the identity element under tensor product. Thus we have

Corollary 2*. Let $A$ be a bialgebra and let $V$ be an A-module. If $\operatorname{ann}_{A} V$ contains no nonzero bi-ideal, then $\mathscr{T}^{*}(V)=\oplus \sum_{n=0}^{\infty} V^{\otimes n}$ is a faithful A-module.

Let $V$ be an $A$-module. If $J$ is an ideal of $A$ contained in $\operatorname{ann}_{A} V$, then we can think of $V$ as having been lifted from an $A / J$-module. In particular, $V$ is faithful if and only if it is not lifted from any proper homomorphic image of $A$. Similarly, if $A$ is a b-algebra, we might say that $V$ is b-faithful if it is 
not lifted from any proper b-algebra homomorphic image of $A$. In other words, $V$ is b-faithful if and only if $\operatorname{ann}_{A} V$ contains no nonzero b-ideal of $A$. Thus Corollary 2 asserts that any b-faithful module $V$ gives rise to the faithful tensor module $\mathscr{T}(V)$. This is essentially Burnside's Theorem.

Let us look at some examples. To start with, recall that a multiplicative semigroup $G$ is a set having an associative multiplication and an identity element 1 . Semigroups may contain a zero element $0 \neq 1$ satisfying $0 g=g 0=0$ for all $g \in G$, and as usual we let $G^{\#}=G \backslash\{0\}$ denote the set of nonzero elements of $G$. The semigroup algebra $K[G]$ is then a $K$-vector space with basis $G^{\#}$ and with multiplication inherited from that of $G$. Notice that the zero element of $G$, if it exists, is identified with the zero element of $K[G]$. Furthermore, $K[G]$ is a b-algebra with $\Delta$ defined by $\Delta(g)=g \otimes g$ for all $g \in G^{\#}$. Given this comultiplication, it is easy to see that the only possible bialgebra structure on $K[G]$ would have counit $\epsilon$ given by $\epsilon(g)=1$ for all $g \in G^{\#}$. But then, $\epsilon$ is an algebra homomorphism if and only if $G^{\#}$ is multiplicatively closed, or equivalently if and only if there are no zero divisors in $G$. In other words, most semigroup algebras are just not bialgebras in this way.

If $H$ is also a semigroup, then a semigroup homomorphism $\theta: G \rightarrow H$ preserves the multiplication and, by definition, it satifies $\theta(1)=1$ and $\theta(0)=0$ if $G$ has a zero element. In particular, it follows that $\theta$ extends to a $K$-algebra homomorphism $\bar{\theta}: K[G] \rightarrow K[H]$ which is clearly a b-algebra map. Hence $\operatorname{ker} \bar{\theta}$ is a b-ideal of $K[G]$. As is well known, these are the only possible b-ideals. Since the argument is so simple, we briefly sketch it here.

Let $I$ be a b-ideal of $K[G]$ and let $\bar{\phi}$ be the b-algebra epimorphism defined by $\bar{\phi}: K[G] \rightarrow K[G] / I=C$. Then $H=\bar{\phi}(G)$ is a multiplicative subsemigroup of $C$ and, since $\bar{\phi}$ is a b-algebra homomorphism, it is easy to see that $H$ consists of group-like elements. In particular, it follows from [Sw, Proposition 3.2.1(b)] that $H^{\#}$ is a linearly independent subset of $C$. Furthermore, since $G^{\#}$ spans $K[G]$, we know that $H^{\#}$ spans $C$. Thus it is clear that $C=K[H]$ and that the map $\bar{\phi}: K[G] \rightarrow K[H]$ is the natural extension of the semigroup epimorphism $\phi: G \rightarrow H$, namely, the restriction of $\bar{\phi}$ to $G$. Since $I=\operatorname{ker} \bar{\phi}$, this fact is proved.

By combining the above with Corollary 2, we can quickly obtain Steinberg's generalization of the classical result of Burnside [B, §226]. The original Burnside theorem concerned modules for the complex group algebra $\mathbb{C}[G]$ with $|G|<\infty$, and the proof used the character theory of finite groups. The argument in [St] is more transparent and, of course, it is more general. But the following proof, due to Rieffel in [Ri], shows precisely why the $G$-faithfulness assumption on the $K[G]$-module $V$ is both natural and relevant.

Let $G$ be a semigroup and let $V$ be a $K[G]$-module. We say that $G$ acts faithfully on $V$ if for all distinct $g_{1}, g_{2} \in G$ we have $\left(g_{1}-g_{2}\right) V \neq 0$. Of course, if $G$ is a group, then this condition is equivalent to $(g-1) V \neq 0$ for all $1 \neq g \in G$.

Theorem 3. Let $G$ be a semigroup and let $G$ act faithfully on the $K[G]-$ module $V$. Then $K[G]$ acts faithfully on the tensor module $\mathscr{T}(V)=\oplus \sum_{n=1}^{\infty} V^{\otimes n}$.

Proof. Let $I$ be a b-ideal of $K[G]$ contained in $\operatorname{ann}_{K[G]} V$. As we observed, there exists a semigroup epimorphism $\phi: G \rightarrow H$ such that $I$ is the kernel of the corresponding algebra map $\bar{\phi}: K[G] \rightarrow K[H]$. If $I \neq 0$, then $\phi$ cannot be 
one-to-one on $G$ and hence there exist distinct $g_{1}, g_{2} \in G$ with $\bar{\phi}\left(g_{1}-g_{2}\right)=0$. In particular, this implies that $g_{1}-g_{2} \in I$, so $\left(g_{1}-g_{2}\right) V=0$, contradicting the fact that $G$ is faithful on $V$. In other words, the $G$-faithfulness assumption implies that $\operatorname{ann}_{K[G]} V$ contains no nonzero b-ideal. Corollary 2 now yields the result.

An analogous result holds for enveloping algebras. For simplicity of notation, let us assume that either

(1) $K$ is a field of characteristic $0, L$ is a Lie algebra over $K$, and $U(L)$ is its enveloping algebra, or

(2) $K$ has characteristic $p>0, L$ is a restricted Lie algebra over $K$, and $U(L)$ is its restricted enveloping algebra.

In either case, $U(L)$ is a b-algebra, and in fact a Hopf algebra, with comultiplication determined by $\Delta(\ell)=\ell \otimes 1+1 \otimes \ell$ for all $\ell \in L$. Furthermore, if $H$ is a second (restricted) Lie algebra and if $\theta: L \rightarrow H$ is a (restricted) Lie algebra homomorphism, then $\theta$ extends uniquely to a b-algebra homomorphism $\bar{\theta}: U(L) \rightarrow U(H)$. In particular, $\operatorname{ker} \bar{\theta}$ is a b-ideal of $U(L)$. As is well known, the converse is also true, namely, every b-ideal of $U(L)$ arises in this manner. The argument for this is elementary and similar to the one for semigroup rings. A sketch of the proof is as follows.

Let $I$ be a b-ideal of $U(L)$ and let $\bar{\phi}$ be the b-algebra epimorphism defined by $\bar{\phi}: U(L) \rightarrow U(L) / I=C$. Then $H=\bar{\phi}(L)$ is a (restricted) Lie subalgebra of $C$ and $H$ generates $C$ as a $K$-algebra. In particular, if $\left\{h_{i} \mid i \in \mathscr{I}\right\}$ is a basis for $H$, indexed by the ordered set $(\mathscr{I}, \prec)$, then $C$ is spanned by monomials of the form $h_{i_{1}}^{e_{1}} h_{i_{2}}^{e_{2}} \cdots h_{i_{n}}^{e_{n}}$ with $i_{1} \prec i_{2} \prec \cdots \prec i_{n}$ and with integers $e_{j} \geq 0$. Furthermore, when char $K=p>0$ and $L$ is restricted, then $e_{j}<p$ for all $j$. Since $\bar{\phi}$ is a b-algebra epimorphism, it follows that the elements of $H$ are primitive. Thus, by the work of [Sw, Chapter 13], these straightened monomials are $K$-linearly independent and therefore $C=U(H)$. In other words, the map $\bar{\phi}: U(L) \rightarrow U(H)$ is the natural extension of the (restricted) Lie algebra epimorphism $\phi: L \rightarrow H$ where, of course, $\phi$ is the restriction of $\bar{\phi}$ to $L$. Since $I=\operatorname{ker} \bar{\phi}$, this fact is proved.

Now let $V$ be a $U(L)$-module. We say that $L$ acts faithfully on $V$ if, for all $0 \neq \ell \in L$, we have $\ell V \neq 0$. The Lie algebra analog of the preceding result is then

Theorem 4. Let $U(L)$ be a (restricted) enveloping algebra satisfying (1) or (2) above. If $L$ acts faithfully on the $U(L)$-module $V$, then $U(L)$ acts faithfully on the tensor module $\mathscr{T}(V)=\oplus \sum_{n=1}^{\infty} V^{\otimes n}$.

As indicated in [M], a theorem of this nature can be used to prove the following interesting result of Harish-Chandra [H, Theorem 1]. Recall that a $K$ algebra $A$ is residually finite if the collection of its ideals $I$ of finite codimension has intersection equal to 0 . In other words, these algebras are precisely the subdirect products of finite-dimensional $K$-algebras.

Corollary 5. If $L$ is a finite-dimensional Lie algebra over a field $K$ of characteristic 0 , then $U(L)$ is residually finite.

Proof. By Ado's theorem (see [J, §VI.2]), $A=U(L)$ has a finite $K$-dimensional module $V$ on which $L$ acts faithfully. Thus, the preceding theorem implies 
that $0=\operatorname{ann}_{A} \mathscr{T}(V)=\bigcap_{n=1}^{\infty} I_{n}$, where $I_{n}=\operatorname{ann}_{A} V^{\otimes n}$. But each $V^{\otimes n}$ is a finite-dimensional $A$-module, so $I_{n}=\mathrm{ann}_{A} V^{\otimes n}$ is an ideal of $A$ of finite codimension, and the result follows.

If $L$ is a finite-dimensional restricted Lie algebra, then its restricted enveloping algebra $U(L)$ is also finite dimensional. Thus the characteristic $p>0$ analog of the above is trivial. On the other hand, infinite-dimensional analogs in all characteristics are obtained in [M].

In the remainder of this paper we will restrict our attention to finite-dimensional Hopf algebras. To start with, a Hopf algebra $(A, \Delta, \epsilon, S)$ is a bialgebra with antipode $S: A \rightarrow A$, and a Hopf ideal is the kernel of a Hopf algebra homomorphism. It is easy to see that $I \triangleleft A$ is a Hopf ideal if and only if it is a bideal with $\epsilon(I)=0$ and $S(I) \subseteq I$. Similarly, a $K$-subalgebra $B$ of $A$ is a Hopf subalgebra if and only if it is a b-subalgebra which is closed under the antipode $S$. Of course, the b-subalgebra condition means that $\Delta(B) \subseteq B \otimes B$. The following is a special case of a surprising result due to Nichols [N, Theorem 1 ]. A simple proof of the subalgebra case can also be found in [Ra, Lemma 1].

Lemma 6. If $A$ is a finite-dimensional Hopf algebra, then any b-subalgebra of $A$ is a Hopf subalgebra and any b-ideal of $A$ is a Hopf ideal of $A$.

Proof. Let $B$ denote either a b-subalgebra of $A$ or a b-ideal of $A$. Furthermore, let $E=\operatorname{Hom}_{K}(A, A)$ be the convolution algebra of $A$ and set

$$
F=\{f \in E \mid f(B) \subseteq B\} .
$$

Certainly $F$ is a $K$-subspace of $E$ and, in fact, $F$ is closed under convolution multiplication. To see the latter, let $f, g \in F$. If $B$ is a b-subalgebra of $A$, then $\Delta(B) \subseteq B \otimes B$ implies that

$$
(f * g)(B) \subseteq f(B) g(B) \subseteq B^{2}=B .
$$

On the other hand, if $B$ is a b-ideal of $A$, then $\Delta(B) \subseteq A \otimes B+B \otimes A$ implies that

$$
(f * g)(B) \subseteq f(A) g(B)+f(B) g(A) \subseteq A B+B A=B
$$

since $B \triangleleft A$.

Now observe that the identity map Id is contained in $F$. Thus, by the above, $F$ contains the convolution powers $\mathrm{Id}^{* n}$ of Id for all $n>0$. Furthermore, since $A$ is finite dimensional, $E$ is also finite dimensional and hence the map Id is algebraic over $K$. In particular, for some $m \geq 1$, we can write $\operatorname{Id}^{* m}$ as a finite $K$-linear combination of the powers $\operatorname{Id}^{* i}$ with $i>m$. But Id has convolution inverse $S$, so by multiplying the expression for $\operatorname{Id}^{* m}$ by $S^{* m}$ and by $S^{*(m+1)}$ in turn, we deduce first that $\epsilon=\operatorname{Id}^{* 0} \in F$ and then that $S=\operatorname{Id}^{*(-1)} \in F$. In other words, $\epsilon(B) \subseteq B$ and $S(B) \subseteq B$.

Finally, if $B$ is a b-subalgebra of $A$, then $S(B) \subseteq B$ implies that $B$ is a Hopf subalgebra. On the other hand, if $B$ is a b-ideal of $A$, then $\epsilon(B) \subseteq B \cap K=0$. Thus, since $S(B) \subseteq B$, we conclude that $B$ is a Hopf ideal of $A$.

The preceding result is false in general for infinite-dimensional Hopf algebras. Some rather complicated counterexamples appear in [N]. 
Theorem 7. Let $A$ be a finite-dimensional Hopf algebra.

(i) If $\mathscr{F}$ is a family of A-modules which is closed under tensor product, then $\bigcap_{V \in \mathscr{F}} \operatorname{ann}_{A} V$ is a Hopf ideal of $A$.

(ii) Suppose $V$ is an A-module whose annihilator contains no nonzero Hopf ideal of $A$. Then $\mathscr{T}(V)=\oplus \sum_{n=1}^{\infty} V^{\otimes n}$ is a faithful A-module.

This follows immediately from Proposition 1, Corollary 2, and Lemma 6 . We can now obtain some consequences of interest. First, recall that an $A$-module $V$ is semisimple if it is a direct sum of simple modules.

Corollary 8. If $A$ is a finite-dimensional Hopf algebra, then the set of semisimple $A$-modules is closed under tensor product if and only if the Jacobson radical $J(A)$ is a Hopf ideal of $A$.

Proof. Let $\mathscr{F}$ be the set of all semisimple $A$-modules. If $\mathscr{F}$ is closed under $\otimes$, then Theorem 7(i) implies that $J(A)=\bigcap_{V \in \mathscr{F}} \operatorname{ann}_{A} V$ is a Hopf ideal of $A$. Conversely, if $J(A)$ is a Hopf ideal, then $\mathscr{F}$ consists of all the modules for the Hopf algebra $A / J(A)$ and therefore $\mathscr{F}$ is surely closed under tensor product.

In a similar manner, we prove

Corollary 9. Let $A$ be a finite-dimensional semisimple Hopf algebra and let $\mathscr{I}$ be a family of simple A-modules. Suppose that, for all $V, W \in \mathscr{I}$, every irreducible submodule of $V \otimes W$ is contained in $\mathscr{I}$. Then $I=\bigcap_{V \in \mathcal{I}} \operatorname{ann}_{A} V$ is a Hopf ideal of $A$ and $\mathscr{I}$ is the set of all simple A/I-modules.

Proof. Let $\mathscr{F}$ be the set of all finite direct sums (with multiplicities) of elements of $\mathscr{I}$. Since $A$ is semisimple, the hypothesis implies that $\mathscr{F}$ is closed under tensor product. Hence, by Theorem 7(i), $I=\bigcap_{V \in \mathscr{I}} \operatorname{ann}_{A} V=\bigcap_{W \in \mathscr{F}} \operatorname{ann}_{A} W$ is a Hopf ideal of $A$. Furthermore, since $A / I$ is semisimple, it follows that $\mathscr{I}$ must be the set of all simple $A / I$-modules.

Our final consequence uses the fact that any finite-dimensional Hopf algebra $A$ is a Frobenius algebra [LS, $\S 5]$ and hence that every simple $A$-module is isomorphic to a minimal left ideal of $A$.

Corollary 10. Let $A$ be a finite-dimensional Hopf algebra and let $V$ be an $A$ module whose annihilator contains no nonzero Hopf ideal of $A$. Then every simple A-module is isomorphic to a submodule of $V^{\otimes n}$ for some $n \geq 1$.

Proof. It follows from Theorem 7(ii) that $\mathscr{T}(V)=\oplus \sum_{n=1}^{\infty} V^{\otimes n}$ is a faithful $A$-module. Now let $W$ be a simple $A$-module, so that $W$ is isomorphic to a minimal left ideal $L \subseteq A$. Since $L \neq 0$, we have $L \mathscr{T}(V) \neq 0$ and hence $L V^{\otimes n} \neq 0$ for some $n \geq 1$. In particular, there exists $u \in V^{\otimes n}$ with $L u \neq$ 0 . But then the minimality of $L$ implies that $W \cong L \cong L u \subseteq V^{\otimes n}$, as required.

\section{ACKNOWLEDGMENT}

In closing, the authors would like to thank S. Montgomery and M. Cohen for informing us about references [M] and [Ri], respectively. 


\section{REFERENCES}

[B] W. Burnside, Theory of groups of finite order (reprint of 2nd edition, 1911), Dover, New York, 1955.

[H] Harish-Chandra, On representations of Lie algebras, Ann. of Math. (2) 50 (1949), 900-915.

[J] N. Jacobson, Lie algebras, Interscience, New York, 1962.

[LS] R. G. Larson and M. E. Sweedler, An associative orthogonal bilinear form for Hopf algebras, Amer. J. Math. 91 (1969), 75-94.

[M] W. Michaelis, Properness of Lie algebras and enveloping algebras I, Proc. Amer. Math. Soc. 101 (1987), 17-23.

[N] W. D. Nichols, Quotients of Hopf algebras, Comm. Algebra 6 (1978), 1789-1800.

[Ra] D. E. Radford, On the quasitriangular structure of a semisimple Hopf algebra, J. Algebra 141 (1991), 354-358.

[Ri] M. A. Rieffel, Burnside's theorem for representations of Hopf algebras, J. Algebra 6 (1967), 123-130.

[St] R. Steinberg, Complete sets of representations of algebras, Proc. Amer. Math. Soc. 13 (1962), 746-747.

[Sw] M. E. Sweedler, Hopf Algebras, Benjamin, New York, 1969.

Department of Mathematics, University of Wisconsin-Madison, Madison, Wisconsin 53706

E-mail address: passman@math.wisc.edu

Department of Mathematics, Syracuse University, Syracuse, New York 13244

E-mail address: dpquinn@mailbox.syr.edu 